\title{
Mapping the Scope of Software Interventions for Moderate Internet Use on Mobile Devices
}

\author{
Joshua Hill, Kelly Widdicks, and Mike Hazas \\ School of Computing and Communications, Lancaster University, Lancaster, UK
}

\{j.hill8, k.v.widdicks, m. hazas\}@lancaster.ac.uk

\begin{abstract}
ICT is expected to form $21 \%$ of global electricity demand in 2030 , and history has shown that efficiency gains in Internet infrastructure aiming to curtail such impacts are far outstripped by the growth in data traffic. We need to reduce demand for Internet connectivity, yet encouraging moderate interactions with digital devices and online services could potentially benefit users. HCI designs have been suggested for moderate interactions and Internet usage, most commonly on smartphones-but it's currently unclear whether these interventions can actually be implemented and tested to understand the user and environmental impacts. In this paper, we review features for understanding and manipulating data traffic in accordance with the stock Android and iOS development libraries to better scope the potential for implementing moderate and sustainable digital experiences. Specifically, we outline the intervention features plausible for Android implementation, and we provide reasoning for why iOS is currently too restrictive. We hope our analysis will break down barriers for researchers interested in this work, or make it easier for them to consider sustainability in their own device or service interventions. We also discuss opportunities for better data traffic consideration in mobile operating systems by 2030 .
\end{abstract}

\section{CCS CONCEPTS}

- Human-centered computing $\rightarrow$ Ubiquitous and mobile devices; • Software and its engineering;

\section{KEYWORDS}

Internet use, data demand, sustainability, everyday life, Internet moderations, digital experiences, digital wellbeing, Android, iOS

\section{ACM Reference Format:}

Joshua Hill, Kelly Widdicks, and Mike Hazas. 2020. Mapping the Scope of Software Interventions for Moderate Internet Use on Mobile Devices. In 7th International Conference on ICT for Sustainability (ICT4S2020), fune 21-26, 2020, Bristol, United Kingdom. ACM, New York, NY, USA, 9 pages. https://doi.org/10.1145/3401335.3401361

\section{INTRODUCTION}

The development and adoption of Internet-connected devices and services enable many positive actions for people in their everyday lives-making activities, such as communicating with family or friends across the world, more frequent and potentially richer

Permission to make digital or hard copies of part or all of this work for personal or classroom use is granted without fee provided that copies are not made or distributed for profit or commercial advantage and that copies bear this notice and the full citation on the first page. Copyrights for third-party components of this work must be honored

For all other uses, contact the owner/author(s).

ICT4S2020, June 21-26, 2020, Bristol, United Kingdom

(C) 2020 Copyright held by the owner/author(s).

ACM ISBN 978-1-4503-7595-5/20/06.

https://doi .org/10.1145/3401335.3401361 through pictures and video. Yet there are increasing concerns about the negative impacts that these services and devices have on the very users that access these technologies, such as issues of addiction and mental wellbeing $[2,5,11,12,93]$. Coinciding with these are environmental concerns over the rate of growth in Internet connectivity and the resources needed to power the Internet infrastructure, with ICT expected to grow to about $21 \%$ of our global electricity consumption by 2030 [3].

Energy efficiencies in the Internet infrastructure are required to prevent the expected trajectories in Internet growth, but these follow a reinforcement feedback cycle whereby: efficiencies in the infrastructure allow for new data-intensive services to be designed, which drives demand for such services, and ultimately leads to further growth and required efficiencies in the infrastructure [73]. Instead, we need to ensure that the demand for Internet connectivity does not continue to eliminate the energy savings from improved efficiency. In 2019, it was positioned to the 'Computing within Limits' community that Human-Computer Interaction (HCI) designs promoting 'moderate' Internet use could be a positive mechanism for reducing the demand for data and encouraging more sustainable use of devices and services [97]. Widdicks and Pargman specifically point out how reductions in Internet data could be embedded within research areas aiming to improve users' digital wellbeing, work productivity, online privacy and relationships with others [97].

The need to decrease the demand for Internet data and use is particularly relevant for smartphones. The traffic consumption from these devices is expected to grow, increasing 10 times for mobile data between 2016-2022 [36] and forming 50\% of total global Internet traffic by 2022 (growing from $23 \%$ in 2017) [16]. Smartphones are also the most commonly used device composing $68 \%$ of the total time people spend online, with mobile devices forming $75 \%$ of time online once tablets are considered [63]. But given our prior knowledge of attempting Internet traffic logging on such devices [54, 95], the feasibility of implementing moderate Internet use has been questioned [97]. In fact, a research app previously used for Internet demand analysis on Android devices [95] recently became deprecated [1]-further sparking our concern for the ability to develop future research and systems in this domain.

With this in mind, this paper uncovers the practicable and plausible software features that we might require to create moderate online interactions on smartphones. Through in-depth trawling of mobile operating system (OS) libraries and online developer forums, we provide an overview of what can currently be developed on the Android platform (85.9\% market share in 2017 [85]) and offer an understanding of why Apple's iOS is presently too restrictive for creating moderate Internet use. We hope that this analysis will break down barriers for other HCI researchers (particularly those working on digital wellbeing [13]) to consider Internet demand in their own smartphone usage interventions-at least in the short 
term. Yet, given OSs change often and rapidly, the main purpose of this paper is instead to highlight the challenging nature of creating mobile software interventions. From this, we offer a discussion of the opportunities in this space, and directions for change in the technology industry, in order for moderate Internet use to become possible in future systems of the year 2030 .

\section{BACKGROUND}

In sustainable HCI, there is a growing body of work investigating "data demand", i.e. "the demand for online services and network connectivity" [54], given the environmental impacts of the Internet infrastructure. Researchers have uncovered data demand across mobile devices [54, 95] and for specific demanding activities such as watching [96], as well as the opportunities to reduce traffic consumption in digital service design [73]. Adopting more sustainable $\mathrm{HCI}$ designs can have a great impact given the popularity of a service, e.g. turning off video content for music which is only listened to on YouTube can have a comparable emissions reduction to running a data centre on renewable energy [74].

To continue to reduce data demand in a way which works positively for users, Widdicks and Pargman [97] suggest creating more moderate use of Internet-connected devices and services. They build upon arguments for more positive approaches to sustainability [57], and offer opportunities for embedding data demand reductions through societal research areas investigating digital wellbeing, work productivity, relationships with others and privacy [97]. This was motivated by rising concerns over the societal impacts of our connected lives and the recent drive to encourage users to spend less time online. Large technology corporations have introduced time-based controls (e.g. Apple [60], Google [88], Facebook and Instagram [76]) and applications to block services exist (e.g. Hold [43], Forest [38], StayFocusd [86], Cold Turkey [90])aiming to help users be more focused on their time in the physical world, such as for work or spending quality time with friends. Most recently, these rising concerns have sparked a new field of exploring 'digital wellbeing' in HCI research aiming for more positive experiences with technology $[10,13,79]$.

In these broader areas of $\mathrm{HCI}$ that aim to alleviate the negative impacts of device or service use on users, different interaction interventions have been designed, developed or evaluated to mitigate technology overuse and improve users' self-control [56]. Kim et al. have explored interaction lockout techniques, investigating a voluntary self-interruption tool to block use across multiple devices [48], as well as different strengths of restrictions [49]. Building on this work [48], Tseng et al. investigate a 'negotiable' blocking system for reducing online distractions in the workplace [89]. Through a more pervasive design approach, "frictions" and "micro-boundaries" to prevent "mindless" technology use [18] have been discussed (e.g. a typing task for smartphone unlocking [50]) or utilise nudge theory and negative reinforcement to reduce digital consumption [64].

Some of this prior work actually prototypes and tests interventions to smartphone use $[49,64]$, meaning it is obvious that there are opportunities for developing software interventions on these mobile platforms to adapt users' device and app use. However, our prior knowledge and work in the field of data demand has found manipulating Internet traffic is not quite as straight forward [97]meaning that embedding data reductions into broader themes of $\mathrm{HCI}$ (e.g. digital wellbeing) through moderate Internet use is perhaps more challenging. But how can smartphone interventions currently monitor and limit data traffic, and how can we make it easier for HCI researchers and service designers to take data demand into account going forward? Given the drive for digital wellbeing applications and for devices and services to promote less time online, how can we fully utilise this opportunity to promote more sustainable Internet consumption so that it's less likely for 2030 's technology systems to continue to contribute to the endless cycle of data growth?

In this paper, we begin to unpack these questions in relation to the popular mobile software platforms created by Apple and Google. Utilising our experience of HCI research and data demand, we explore the software features required to understand and manipulate data traffic on mobile operating systems-outlining the possibilities on Android, and providing an explanation of why iOS is currently too restrictive for such developments.

The most relevant prior work in software development has tended to address certain, specific application domains, for example: technical limitations for app design for social media [78]; forensic tools on Android [77]; a quality assessment of current wellbeing apps [19]; the power consumption of applications and particular development methods [20,65]; and developing secure code [61]. There has also been much work exploring the general challenges of app design and creation on mobile platforms [6, 47]. However, none of these address the specific implementation of software which aims to enable more moderate Internet use. Thus, we begin by exploring the current scope of these tools on Android in 2020.

\section{ANDROID SCOPE IN 2020}

In this section, we iterate through six core features that are essential, or potentially useful, for reducing data demand through more moderate Internet use on mobile devices. These features are derived from sampling existing or proposed interventions. For each feature, we explain why it would be relevant in this design space and whether it is plausible to implement it on Android smartphones and tablets-offering workarounds and alternatives where OS restrictions are met. This involved a member of the research team carrying out at least one month of in-depth trawling of API (Application Programming Interfaces) and operating system libraries (e.g. Android Developer), developer forums (e.g. Stack Overflow) and news articles which we cite to back-up our analysis. To the best of our knowledge, the information we provide in this section is up-to-date as of March 2020.

\subsection{Running interventions continuously}

In order for intervention apps to track aspects of data demand, and take action to moderate Internet use, it will likely need to run services in the 'foreground'; this means an intervention can run continuously without being 'killed' by the Android OS attempting to free up resources. An example of such a service is a music player, which wont be stopped by the Android OS if left playing whilst using other applications. Running services in the foreground is required by many mobile interventions which need to take actions 
in 'real time', e.g. for tracking screen time or app usage, logging data throughput in bytes, and employing interventions such as Internet 'speedbumps' [94] (i.e. the purposeful throttling of bandwidth) [cf. $51,54,64,97]$. Without this foreground service, data usage (e.g. data usage per application, see section 3.2) could only be retrieved when the intervention is launched and in focus to perform the data gathering; this launching action is taken by the user, limiting the intervention's ability to understand or automatically moderate Internet use in real time.

To set up foreground service running in Android, the appropriate notification channel needs to be established; this ensures that the user is aware an intervention app is running a service, and prevents the existence of potentially malicious foreground services without the user's consent [31, 32]. Foreground services on Android can be developed as long as they do not use excessive system resources; any loops used should contain code that slows down their execution [31] This means that, if developed efficiently, running an intervention to moderate Internet use continuously is possible and indeed critical to many of their operations.

\subsection{Logging Internet Traffic}

Logging Internet traffic to and from the device is clearly required to understand data demand, allowing apps to be targeted that put strain on the network (e.g. through large updates, many background processes, or high usage from the user themselves) as well as determining if a given intervention reduces data demand successfully. It is possible to record overall throughput for the device using the 'TrafficStats' API, but this no longer provides data use on a per application basis [33]. Instead, a '-1 unsupported' error would be returned for per app data statistics as this access was depreciated in Android 4.1 (JellyBean) due to security issues of revealing transport layer information [33]. However, it is possible to get a breakdown of WiFi or mobile data throughput over a given time period for specific applications, or since their instillation, by combining data from the 'Package Manager' and 'Network Stats Manager' APIs in Android 8 (Oreo). Package Manager can be used to retrieve a list of all installed applications on the device, and its corresponding metadata held in a 'ApplicationInfo' data structure. This data can hence be used to create a breakdown of app process IDs that can be fed into the NetworkStatsManager 'Query Details for UID' method to obtain throughput information on each application [26, 29].

Alternatively, it would be possible to roughly attribute throughput to apps based on the current foreground activity and total Internet usage (e.g. using the TrafficStats API)-but this comes with its own issues of accuracy given apps can demand data in the background (i.e. when the user is not interacting with the app) [33] It is easier to instead investigate foreground vs. background data demand as a whole, logging overall traffic consumption as well as when the user is, or is not, accessing their device (e.g. if the device is on/off or locked/unlocked using the 'isInteractive' call that is part of the 'PowerManager' API) [30, 66]. On the reverse, interventions could infer what type of app is being used based on the amount of data being transferred (e.g. high data volumes would suggest video streaming) and take appropriate moderation action, but this would obviously require some assumptions. Overall, it is currently difficult to gain an accurate reflection of data demand and its potential reduction across apps or activities from moderate Internet use interventions and better, more reliable tools would be required for future work.

\subsection{Logging screen time}

Logging app screen time is important in understanding what apps are used the most and when. App use does not directly correlate to data demand, e.g. data could be demanded whilst the smartphone or application is not in use. However, utilising this knowledge could be useful for uncovering how device use and data demand fits into users' everyday life, as well as to enforce traffic limits or interventions focused on screen time. Such understandings of screen time have been required by device or app intervention designs or prototypes explored in prior work [48, 51, 53, 64, 96]. In Android, it is possible to get a breakdown of screen time per app since installation or over a given time period, the last time used and more through the 'UsageStatsManager' API. UsageStatsManager's 'queryUsageStats' method returns a list of 'UsageStats' data structures, one for each application, which can be queried to retrieve the desired information [34]. It is possible to know when the application was installed, last used and its total time in foreground [29] as seen above, hence we can begin to determine how data demand is linked with screen time by querying periods such as between the current time and an applications last used time to track data demand when the application is not being used.

Unfortunately this method does not offer a 'live' view of usage statistics, as the statistics only update when apps are closed or refreshed; this means that if you were to launch application X for the first time, the usage statistic for application $X$ would stay at 0 until it was closed or refreshed. A workaround for this is to implement your own internal counter running in a foreground service, see section 3.1). This can be achieved by obtaining the current application in focus, detected by checking the foreground activity, and using the OS's built-in time library (introduced in Android 8 (Oreo)) to increase a counter for that application accurately, at least until the UsageStats are updated [7, 24, 31]. Internal counters could be reset at the end of each day for example, or when an application is no longer being moderated. On the same front, Android 9 (Pie) saw the release of Android's official 'Digital Wellbeing' app which tracks time in app and various usage measures (e.g. number of times opened) in far greater detail than previous OS versions offered which holds great potential over current tools-as this app has system level privileges however, the same data will not be available to third party developers despite its potential to greatly ease the creation of new intervention applications $[4,45]$.

\subsection{Toggling WiFi and mobile data settings}

Limiting or disabling access to WiFi and mobile data is one clear way to intervene with data demand-as pointed out by prior work [ 54 , $72,96,97]$. This is particularly relevant for mobile data access (e.g. $3 \mathrm{G}, 4 \mathrm{G}$ ) which is expected to be more energy-intensive than WiFi networks [54]. Whilst Android devices offer features to determine whether they are connected to mobile or WiFi networks [25], throttling bandwidth to limit Internet access speeds is not possible without root access to the device or creating your own private network 
and manually adjusting it $[69,82]$. This means that bandwidth throttling would be required at a lower level (e.g. a household router) to increase loading times on devices or force them into a 'low data mode' $[41,80,96]$. As for disabling WiFi completely, it is possible to programmatically turn WiFi on or off up to Android 9 (Pie) using the 'WifiManager'-but that access has now been revoked for future Android versions, a step backwards for intervention developers [35]. Furthermore, it has not been possible to do the same for mobile data since Android 2.2 (Froyo) [67, 99]. In Android versions before 9 (Pie), it would nonetheless be unethical to turn off WiFi access without also turning off mobile data because users may incur high data usage costs if they do not realise this has happened (a reason for why this software feature is not available in iOS-discussed in section 4).

A possible workaround to the problem of being unable to programmatically toggle mobile data connectivity is to continuously display the mobile data options screen to the user whilst mobile data is still enabled [17,83]-forcing the user to switch mobile data off themselves before being able to access other applications. However, this is obviously an intrusive and forceful approach which will no doubt create a frustrating user experience, and even then would require an option for the user to cancel the intervention in-case access to mobile data was essential. For blocking internet connectivity to specific applications instead of phone-wide, a Virtual Private Network (VPN) application is a possible way forward for moderate Internet use interventions as they would offer far more control over the network [81].

\subsection{Managing notifications and device modes}

OS features provided by Android and Apple offer notification blocking through automatic enabling of 'do not disturb' modes $[4,60]$, and similar interventions to data demand in HCI have been discussed [54, 94]. By manipulating the notification delivery and device modes, there is the expectation that the device will reduce the impact it has on users' attention given notifications can be perceived as disruptive; this may therefore help to create more moderate Internet use for the user and therefore reduce the associated data traffic. Achieving this effect is possible in Android using the 'NotificationManager' and 'AudioManager' features, device modes can be adapted between 'do not disturb' (silent), vibrate and ringer $[22,28]$. The disadvantage to this approach is that changes will apply system-wide, and disabling notifications for specific applications can only currently be done through Android's own OS settings menu [42]-hence it is not possible to create an intervention that toggles notifications for certain applications only.

Google's new digital wellbeing application for Android $10(\mathrm{Q})$ is set to include a 'pause' feature as part of its 'focus mode', which will block access to, and notifications from, specific applications that the user determines as 'distracting' for the rest of the day-greying out the app icon in menus to visually show that the application is currently 'paused' [14, 37, 39]. Although only in its Beta phase, this will be the first system-supported option for users to disable notifications and access to specific applications temporarily, and then have them automatically re-enable. In terms of notifications, this is far better than the 'all or nothing' approach of disabling all notifications for an application in the device settings indefinitely, or blocking notifications from all apps using 'do not disturb' mode [42]. Additionally, the new digital wellbeing application will allow bundling of notifications into a 'digest' for applications such as YouTube so that the user only gets one notification rather than many throughout the day [39]. Given these tools are built-in, they make studying the impact of blocking notifications and certain applications on data demand much easier, and the impact can be understood at a much finer level (e.g. the data demand impact of blocking social media application notifications). Yet again, it seems unlikely these features will be accessible to third party developers, so customisation and refinement of the features offered in subsequent third-party interventions may not be possible.

Permanent notifications are potentially one way in which an intervention could highlight to a user how much time they spend on their device or how much data they are using [94]. These can be created using the foreground service notification channel or by implementing an 'ongoing' notification which cannot be dismissed by the user [27, 32]. Showing current screen time, data traffic consumption for certain applications, or total daily throughput for the device in this way would be highly visible-requiring less motivation for the user to access this information if it was hidden within another application, making it a powerful tool for interventions to harness.

\subsection{Measuring and managing scrolling}

Measuring gesture scrolling has been discussed as one way to determine whether users are engaging with the content they are consuming [94]. This measurement of scrolling has been carried out by prior work [55] and can be tracked using the 'GestureDetector' [23]. Gaining information from this gesture interaction is tricky, however. The 'distance' and speed in which the user has scrolled can be approximated, and the application in which the user was scrolling through can be estimated given the findings in section 3.3but it will be difficult to determine what content exactly initiates users to carry out such scrolls. Qualitative research with users (e.g. interviews) would help fill this missing knowledge gap, but this would not help interventions which utilise 'automatic scrolling' to slow down users' interactions [94]. An additional challenge is that apps such as Facebook and Instagram offer auto-scrolling (e.g. in the form of auto-playing the next video), requiring no input from the user to view the next content or 'post'; this would effectively bypass scrolling measurement given they drive the user to consume more content (and therefore more data) rather than less.

\section{IOS SCOPE IN 2020}

Unlike Android's open source nature [9], Apple is much more restrictive in their iOS runtime and App Store policy. Our iOS development attempts, and research on developer websites and forums, indicated that many of the potential interventions for moderate Internet use and reduction of data demand cannot be developed for Apple mobile devices. For example, Internet access (e.g. through WiFi toggling) cannot be manipulated as WiFi is a global system setting that cannot be accessed by third party apps. There are claims that this restriction is due to fears that malicious apps may enforce mobile data access by toggling WiFi off, potentially causing the 
device to surpass its mobile data limits thus causing loss of service or additional expense [75, 84]. Understanding screen time for apps is also difficult, with the most common methods of using Mobile Device Management (MDM) or VPN technology becoming more restricted by Apple; this is due to concerns that gaining this information uses invasive techniques that could put users' data at risk [71, 87]. Other restrictions abound: notifications cannot be turned on or off automatically; 'do not disturb' mode cannot be changed directly by apps; automatically launching apps from the home screen is not allowed; websites can only be blocked through Apple's Safari browser; and a list of running app IDs cannot be collected without jail-breaking or using private frameworks. These restrictions therefore make it difficult to do any app-specific data gathering or interventions of Internet or app use [68, 70].

Perhaps the most important feature for online service interventions is the ability to log data demand in the background to understand if the moderation of Internet use makes any tangible reduction of data use. However, apps require special permission to run in the background (e.g. for background fetch, audio VoIP or Bluetooth, or location updates [21]), subsequently meaning background logging apps would be rejected from the Apple App Store [97]. These factors highlight how iOS is significantly more restrictive than Android, causing a certain company's wellbeing app to have different functionality on the iOS and Android platforms [70]. Further, Apple recently removed many digital wellbeing apps from their App Store-arguing that they breached app store policies. This has resulted in speculations of Apple dominating specific features from other app developers [62, 71, 87], with developers feeling that the iOS development environment is too limited and leading them to create petitions to open up the iOS APIs $[15,70]$.

Some moderate Internet use intervention features (e.g. continuous run permissions) on iOS may be developed and manually installed on iPhones locally (similar to approaches taken by Lord et al. [54] and Gordon et al. [40]) to avoid App Store checks and restrictions. However, accessing other features and deploying remotely at a large scale would require jailbreaking the devices; this approach has been taken in order to conduct large scale iPhone usage studies [59]. Such restrictions suggest why investigations of iOS use are relatively scarce in comparison to Android usage studies [e.g. 8, 46, 92, 100].

\section{DISCUSSION: FROM NOW TO 2030}

Based on both the Android and iOS analysis, it is possible to introduce some interventions for moderate Internet use on mobile devices. However, there are complexities. Whilst Android offers more functionality than iOS, it is still difficult to gather a complete picture of data demand (section 3.2) and the functionality to manipulate it (section 3.4), especially with the constantly changing landscape of allowed APIs and policies. In order for data demand reductions discussed in sustainable HCI to be easily adopted by researchers across HCI (e.g. in digital wellbeing), as well as for data traffic to be more explicitly considered going forward, we now discuss two illustrative pathways for mobile OS designers and developers: option 1) building in more support within the operating system, where the OS would expose analytics but retain control of data traffic monitoring and manipulation; or option 2) opening up to the community, whereby data traffic monitoring and manipulation is made more accessible to app developers. In both cases, these obviously come with the caveat of requiring the OS companies' cooperation; if not, regulatory enforcement (e.g. through technology policy) may be required. However, we hope that, through these options, mobile OS and app developers will begin to explore data demand reductions through more moderate Internet use, and that the community will be more equipped for ensuring sustainable data traffic in 2030 .

\subsection{Option 1: Building in more support}

As Android and iOS have developed over the past decade, the broad trend has been to close down the ability for apps to monitor other apps' cellular and WiFi network traffic volume, screen time, and CPU (Central Processing Unit) time; all three of these are useful proxies for estimating the resources needed to support an app, from within the device, and across the network and data centre. Privacy reasons have most often been used to justify removing apps' ability to monitor one another. This has affected some battery and CPU profiling apps, and also significantly affected apps used to manage and reflect upon screen time, or address addictive behaviour. ${ }^{1}$ These have been replaced with built-in functions like the 'Battery' and 'Screen Time' sections of iOS's Settings.

However, the removed ability to monitor per-app network traffic volume has not been replaced in such a way. In this area, there is some promise for iOS, through the 'Low Data Mode'. The explanatory text for this reads, "Low Data Mode helps reduce Wi-Fi and mobile data usage. When Low Data Mode is turned on, automatic updates and background tasks, such as Photos syncing, are paused." But iOS and Android need to go much further to support energyconscious usage of data-intensive online services. Similar to 'Screen Time', a 'Data Traffic' section could be added to the OS Settings to allow reflection for more moderate Internet use. Here, users could look at the per-app attribution of traffic volume, and perhaps even inspect how much of each app's data was auto-loaded (e.g. delivery of adverts or auto-play of videos on social networking sites), as opposed to resulting from user actions. As with 'Battery', the user might be able to view the traffic volume for the past 24 hours, and daily averages for the past week or month. It might be possible to throttle traffic for given applications, allowing users to find the data rates that support what they want to do, without the app gorging on data. And finally, the user would be able to set preferences for specific types of traffic: video and audio streaming could be set to lower rather than the highest resolutions; OS updates could be set to not download until requested; updates could be set to be once a month per app, maximum; and cloud backups of photos could be set to be lower resolution ones. Throttling, updates and resolution controls could all be set automatically with an 'Eco Data' mode switch at the top of the 'Data Traffic' section.

\subsection{Option 2: Opening up to the community}

Option 2 for iOS and Android mobile operating systems-taking a much more open-source approach-is allowing other mobile platform developers to create their own moderate Internet use interventions by 2030. Android already offers some functionality for

\footnotetext{
${ }^{1}$ https://www.nytimes.com/2019/04/27/technology/apple-screen-time-trackers.html
} 
this software, and is in fact forcing Android mobile phone manufacturers (e.g. Sony, Samsung) to create digital wellbeing apps in Android 9 that fit to their standards [58]; this is a positive move given there is potential to reduce data demand through these digital wellbeing tools [97]. However, there is still movement required for both iOS and Android to make it easier to develop in this space by: 1) improving API capability transparency; 2) enabling Internet manipulations; and 3) creating permissions to collect data usage.

5.2.1 API capability transparency. By 2030, mobile platform developers need to make the capabilities of their APIs more transparent. Whilst there is an abundance of information online for developing services (e.g. on the developer libraries, programming forums), finding out whether specific Internet intervention features are possible is still incredibly time-consuming and complex. The analysis in this paper alone took at least one working month of researcher time, which highlights the time potentially wasted for many developers working in this space. Instead of primarily focusing on specific library functions and relying on websites such as Stack Overflow to help decipher whether a moderate Internet use intervention feature is possible, we position that mobile platform developers should also offer information about what features can or cannot be created. Of course, this is difficult to achieve for all uses of mobile operating systems, but this could easily be introduced for the development of digital wellbeing tools. Furthermore, if a feature isn't available, such information on why this is the case should also be made clear to developers-improving the transparency of the platform and avoiding potentially negative responses towards it (e.g. as with iOS and their digital wellbeing restrictions $[15,70]$ ). This would all save time for developers, researchers, and create a positive development environment for the community.

5.2.2 Internet manipulations. In order to embed data demand reductions within moderate Internet use interventions by 2030, we need to ensure that iOS and Android operating systems allow more mechanisms for manipulating Internet access, traffic and speed. This is particularly required across different apps, so that we can create more tailored approaches for users. However, we do not suggest tailoring by access type: we should treat WiFi and mobile data intervention features as one, removing the concern of users incurring high data costs (as discussed in our analysis, sections 3.4 and 4). Despite the additional data required to track to manipulate traffic and the associated privacy concerns, this can actually utilised to enhance users' privacy if used ethically. For example, data traffic from apps at particular times and to different domains could be visualised to users to better highlight what apps do in the 'background' [94]-building on work that aims to raise users' awareness of their online data use and privacy [52, 91, 98]. Furthermore, such transparency and facilities to fully turn Internet access off for certain apps would enable users to have more control over when data is shared-particularly as this could be their personal data used for data analytics and advertising.

5.2.3 Accessing permissions to collect data usage. If option 2 was to be considered by companies such as Google and Apple, there will of course be security trade-offs. There could be privacy implications surrounding collecting Internet traffic and also potentially negative ways that Internet access, traffic and speed could be manipulated.
For example, a company app could throttle the bandwidth for their competition's app and drive away their users. Internet traffic data could also be used to further understand users through data analytics and manipulate adverts accordingly to drive user consumption. Given this, we note that mobile operating systems in 2030 will need to include additional permissions to access these features for moderate Internet use interventions. Similar to how iOS apps require permissions to continuous background execution from Apple, developers in this space would require special permissions to access and manipulate Internet traffic. This would put additional work on mobile platform providers such as Apple and Google to assess whether an app should have access to this feature, and perhaps this would also require regulation to ensure mobile OS providers themselves use this accordingly (e.g. through additions to the General Data Protection Regulation (GDPR) [44]); but we see this as the only way forward to ensure ethical use of these features for more sustainable, moderate Internet use.

\section{CONCLUSION}

In this paper, we have analysed developer websites and forums to uncover the plausibility of developing moderate Internet use interventions for reducing data demand on iOS and Android. Specifically, we have delved into detail on what features can be explored on Android and offered an explanation of how iOS is currently too restrictive in this design space. Given the complexities of this work, we have offered two ways forward for mobile operating providers: 1) to continue as a closed system, leaving the control for embedding data demand reductions in the hands of companies such as Google and Apple; or 2) to make this space more open-source, ensuring clear and ethical use of moderate Internet use interventions through more transparent API information, Internet manipulation features, and access permissions. We hope that our analysis will break down barriers for researchers working in moderating digital technology use (e.g. in digital wellbeing) to consider data demand in their interventions, and more importantly, that mobile operating systems will begin to work towards ensuring more sustainable data traffic on their platforms by 2030 .

\section{ACKNOWLEDGEMENTS}

We thank the EPSRC for their financial support (grant numbers W95738G and EP/R513076/1).

\section{REFERENCES}

[1] Device Analyzer, 2020. https://deviceanalyzer.cl.cam.ac.uk/, accessed January 2020 .

[2] Hilary Andersson. Social media apps are 'deliberately' addictive to users. $B B C$ News, 2018. https://www.bbc.co.uk/news/technology-44640959, accessed January 2020

[3] Anders S. G. Andrae and Tomas Edler. On global electricity usage of communication technology: Trends to 2030. Challenges, 6(1):117-157, 2015.

[4] Android. Digital wellbeing: New ways to find balance for you and your family, 2020. https://www. android.com/digital-wellbeing/, accessed February 2020.

[5] The BBC. WHO: Gaming addiction 'a mental health condition'. $B B C$ News, 2018. https://www.bbc.co.uk/news/av/uk-44504683/whogaming-addiction-a-mental-health-condition/, accessed February 2020 .

[6] S. Beyer and M. Pinzger. A manual categorization of Android app development issues on stack overflow. In 2014 IEEE International Conference on Software Maintenance and Evolution, pages 531-535, 2014. 
[7] The Lacuna Blog. How to detect foreground application in Android? 2018. https://www. thelacunablog.com/detect-foreground-ap plication-android.html, accessed September 2019.

[8] Matthias Böhmer, Brent Hecht, Johannes Schöning, Antonio Krüger, and Gernot Bauer. Falling asleep with Angry Birds, Facebook and Kindle: A large scale study on mobile application usage. In Proceedings of the 13th International Conference on Human Computer Interaction with Mobile Devices and Services, MobileHCI '11, pages 47-56, New York, NY, USA, 2011. ACM.

[9] M. Butler. Android: Changing the mobile landscape. IEEE Pervasive Computing, 10(1):4-7, Jan 2011

[10] Rafael A. Calvo and Dorian Peters. Design for wellbeing - tools for research, practice and ethics. In Extended Abstracts of the 2019 CHI Conference on Human Factors in Computing Systems, CHI EA '19, pages C15:1-C15:5, New York, NY, USA, 2019. ACM.

[11] Denis Campbell. Children aged five and under at risk of internet addiction - barnardo's. The Guardian, 2019. https://www. theguardian.com/so ciety/2019/jun/11/children-aged-five-and-under-at-riskof-internet-addiction-barnardos, accessed January 2020.

[12] Denis Campbell. Social media firms 'should hand over data amid suicide risk' The Guardian, 2020. https://www. theguardian.com/media/2020/ jan/17/academics-call-for-social-media-data-to-protectyoung-people, accessed January 2020

[13] Marta E. Cecchinato, John Rooksby, Alexis Hiniker, Sean Munson, Kai Lukoff, Luigina Ciolfi, Anja Thieme, and Daniel Harrison. Designing for digital wellbeing: A research \& practice agenda. In Extended Abstracts of the $2019 \mathrm{CH}$ Conference on Human Factors in Computing Systems, CHI EA '19, pages W17:1W17:8, New York, NY, USA, 2019. ACM.

[14] Umang Chamaria. Medium. app pause in Android Q?, 2019 https://medium.com/@umangchamaria/app-pause-in-androidq-285ec752350c, accessed February 2020.

[15] Change.org. Apple: let developers help iPhone users with mental wellbe ing. https://www. change.org/p/apple-allow-digital-wellne ss-developers-to-help-ios-users, accessed Februrary 2020.

[16] Cisco. Cisco Visual Networking Index: Forecast and trends, 2017--2022. Technical report, Cisco, 2019. https://www.cisco.com/c/en/us/so lutions/collateral/service-provider/visual-networkingindex-vni/white-paper-c11-741490.pdf, accessed January 2020

[17] Code Project. by using intent how we got mobile network setting page through in android. https://www.codeproject.com/Questions/568759/by plususingplusintentplushowplusweplusgotplusplusm, accessed February 2020

[18] Anna L Cox, Sandy JJ Gould, Marta E Cecchinato, Ioanna Iacovides, and Ian Renfree. Design frictions for mindful interactions: The case for microboundaries. In Proceedings of the 2016 CHI Conference Extended Abstracts on Human Factors in Computing Systems, pages 1389-1397. ACM, 2016.

[19] Elizabeth Cummings, Elizabeth marie Borychi, and Erin Roehrer. Issues and considerations for healthcare consumers using mobile applications. Enabling Health and Healthcare Through ICT: Available, Tailored and Closer, 2013.

[20] S. K. Datta, C. Bonnet, and N. Nikaein. Android power management: Current and future trends. In 2012 The First IEEE Workshop on Enabling Technologies for Smartphone and Internet of Things (ETSIoT), pages 48-53, 2012.

[21] Apple Developer. UIBackgroundModes, 2020. https://developer .appl e.com/documentation/bundleresources/information_proper ty_list/uibackgroundmodes, accessed February 2020.

[22] Android developers. AudioManager. https://developer.android.com/ reference/android/media/AudioManager, accessed September 2019.

[23] Android developers. GestureDetector. https://developer.android.co $\mathrm{m} / \mathrm{reference/android/view/GestureDetector}$, accessed September 2019.

[24] Android developers. java.time. https://developer.android.com/re ference/java/time/package-summary, accessed September 2019.

[25] Android developers. Monitor connectivity status and connection metering. https://developer . android.com/training/monitoring-de vice-state/connectivity-status-type, accessed September 2019.

[26] Android developers. NetworkStatsManager. https://developer.androi d. com/reference/android/app/usage/NetworkStatsManager, accessed September 2019

[27] Android developers. Notification.Builder: setOngoing. https: //developer.android.com/reference/android/app/Notifi cation.Builder.html\#setOngoing(boolean), accessed February 2020.

[28] Android developers. NotificationManager. https://developer.android. com/reference/android/app/NotificationManager, accessed September 2019 .

[29] Android developers. PackageManager. https://developer.android.co $\mathrm{m} / \mathrm{reference/android/content/pm/PackageManager,} \mathrm{accessed} \mathrm{Sep-}$ tember 2019 .
[30] Android developers. PowerManager. https://developer. android.co $\mathrm{m} / \mathrm{reference/android/os/PowerManager,} \mathrm{accessed} \mathrm{September} 2019$

[31] Android developers. Services overview. https://developer.android. com/guide/components/services, accessed September 2019.

[32] Android developers. Services overview: Running a service in the foreground. https://developer.android.com/guide/components/s ervices.html\#Foreground, accessed September 2019.

[33] Android developers. TrafficStats. https://developer.android.com/re ference/android/net/TrafficStats, accessed September 2019.

[34] Android developers. UsageStatsManager. https://developer.andr oid.com/reference/android/app/usage/UsageStatsManager, accessed September 2019.

[35] Android developers. WifiManager. https://developer.android.co $\mathrm{m} / \mathrm{reference/android/net/wifi/WifiManager,} \mathrm{accessed} \mathrm{September}$ 2019.

[36] Ericsson. Future mobile data usage and traffic growth, 2020. https://www.ericsson.com/en/mobility-report/futuremobile-data-usage-and-traffic-growth, accessed January 2020

[37] Joe Fedewa. Focus mode arrives in the latest digital wellbeing beta. $x d a$ developers, 2019. https://www. xda-developers.com/focus-modedigital-wellbeing/, accessed February 2020.

[38] Forest, 2019. An application for promoting time offline: https://www . fore stapp.cc/, accessed January 2020.

[39] Google. Digital Welbeing: Minimize distractions. https://wellbeing. google/get-started/minimize-distractions/, accessed February 2020 .

[40] Mitchell L. Gordon, Leon Gatys, Carlos Guestrin, Jeffrey P. Bigham, Andrew Trister, and Kayur Patel. App usage predicts cognitive ability in older adults. In Proceedings of the 2019 CHI Conference on Human Factors in Computing Systems, CHI '19, New York, NY, USA, 2019. Association for Computing Machinery.

[41] Tim Hardwick. How to Reduce Your iPhone or iPad Network Data Usage With iOS 13's Low Data Mode. MacRumors, 2019. https://www. macrumors . co m/how-to/use-low-data-mode/, accessed September 2019.

[42] Android Help. Control notifications on Android. https://support.goog le.com/android/answer/9079661, accessed February 2020.

[43] Hold, 2019. An application which rewards users for spending time off their device: https: //www. hold.app/, accessed January 2020.

[44] UK Government: ico. (Information Commissioner's Office). Guide to the General Data Protection Regulation, 2018. https: //www.gov.uk/governm ent/publications/guide-to-the-general-data-protectionregulation, accessed March 2020.

[45] Ivan Jenic. Mobile Internist. how to check screen time in Android Pie, 2019. https://mobileinternist.com/screen-time-android-pie, accessed February 2020

[46] Simon L. Jones, Denzil Ferreira, Simo Hosio, Jorge Goncalves, and Vassilis Kostakos. Revisitation analysis of smartphone app use. In Proceedings of the 2015 ACM International foint Conference on Pervasive and Ubiquitous Computing, UbiComp '15, pages 1197-1208, New York, NY, USA, 2015. ACM.

[47] M. E. Joorabchi, A. Mesbah, and P. Kruchten. Real challenges in mobile app development. In 2013 ACM / IEEE International Symposium on Empirical Software Engineering and Measurement, pages 15-24, 2013.

[48] Jaejeung Kim, Chiwoo Cho, and Uichin Lee. Technology supported behavior restriction for mitigating self-interruptions in multi-device environments. Proc. ACM Interact. Mob. Wearable Ubiquitous Technol., 1(3), September 2017.

[49] Jaejeung Kim, Hayoung Jung, Minsam Ko, and Uichin Lee. Goalkeeper: Exploring interaction lockout mechanisms for regulating smartphone use. Proc. ACM Interact. Mob. Wearable Ubiquitous Technol., 3(1), March 2019.

[50] Jaejeung Kim, Joonyoung Park, Hyunsoo Lee, Minsam Ko, and Uichin Lee. Lockntype: Lockout task intervention for discouraging smartphone app use. In Proceedings of the 2019 CHI Conference on Human Factors in Computing Systems, page 697. ACM, 2019.

[51] Minsam Ko, Subin Yang, Joonwon Lee, Christian Heizmann, Jinyoung Jeong, Uichin Lee, Daehee Shin, Koji Yatani, Junehwa Song, and Kyong-Mee Chung. Nugu: a group-based intervention app for improving self-regulation of limiting smartphone use. In Proceedings of the 18th ACM conference on computer supported cooperative work \& social computing, pages 1235-1245. ACM, 2015.

[52] Marc Langheinrich. Privacy by design - principles of privacy-aware ubiquitous systems. In Ubicomp 2001: Ubiquitous Computing, pages 273-291, Berlin, Heidelberg, 2001. Springer Berlin Heidelberg.

[53] Markus Löchtefeld, Matthias Böhmer, and Lyubomir Ganev. Appdetox: helping users with mobile app addiction. In Proceedings of the 12th international conference on mobile and ubiquitous multimedia, page 43. ACM, 2013.

[54] Carolynne Lord, Mike Hazas, Adrian K. Clear, Oliver Bates, Rosalind Whittam, Janine Morley, and Adrian Friday. Demand in my pocket: Mobile devices and the data connectivity marshalled in support of everyday practice. In Proceedings of the 33rd Annual ACM Conference on Human Factors in Computing Systems, CHI '15, pages 2729-2738, New York, NY, USA, 2015. ACM. 
[55] Li Lu, Jiadi Yu, Yingying Chen, Yanmin Zhu, Minglu Li, and Xiangyu Xu. I3 Sensing scrolling human-computer interactions for intelligent interest inference on smartphones. Proc. ACM Interact. Mob. Wearable Ubiquitous Technol., 3(3), September 2019.

[56] Ulrik Lyngs, Kai Lukoff, Petr Slovak, Reuben Binns, Adam Slack, Michael Inzlicht, Max Van Kleek, and Nigel Shadbolt. Self-control in cyberspace: Applying dual systems theory to a review of digital self-control tools. In Proceedings of the 2019 CHI Conference on Human Factors in Computing Systems, CHI '19, New York, NY, USA, 2019. Association for Computing Machinery.

[57] Samuel Mann, Oliver Bates, Glenys Forsyth, and Phil Osborne. Regenerative computing: De-limiting hope. In Proceedings of the 2018 Workshop on Computing Within Limits, LIMITS '18, pages 1:1-1:10, New York, NY, USA, 2018. ACM

[58] Mariella Moon. Google forces Android phone makers to offer digital wellbeing tools. engadget, 2019. https://www.engadget.com/2019/ 10/08/google-android-wellbeing-requirement/?guce_refe rrer=aHR0cHM6Ly93d3cuZ29vZ2xlLmNvLnVrLw\&guce_referrer sig=AQAAAAXx256cjmELE-GyuZBTSuH707SVBqB2y-pI77GnTAJhw XntANjG1JT3LvPqwWk1Y5z7_4s_bjlNXveRzvgne1BmlUBCb0z7IhbP23zfsGIz69IBNjKu_Mtrom0GRtLg7rtyY8RrHDSSRW8bjY293Rmj YxonRpARU_pkXyowZR0\&guccounter=2, accessed February 2020.

[59] Alistair Morrison, Xiaoyu Xiong, Matthew Higgs, Marek Bell, and Matthew Chalmers. A large-scale study of iPhone app launch behaviour. In Proceedings of the 2018 CHI Conference on Human Factors in Computing Systems, CHI '18, pages 344:1-344:13, New York, NY, USA, 2018. ACM.

[60] Apple Newsroom. iOS 12 introduces new features to reduce interruptions and manage Screen Time, 2018. https://www.apple.com/uk/newsr oom/2018/06/ios-12-introduces-new-features-to-reduceinterruptions-and-manage-screen-time/, accessed January 2020

[61] Duc Cuong Nguyen, Dominik Wermke, Yasemin Acar, Michael Backes, Charles Weir, and Sascha Fahl. A stitch in time: Supporting Android developers in writingsecure code. In Proceedings of the 2017 ACM SIGSAC Conference on Computer and Communications Security, CCS '17, page 1065-1077, New York, NY, USA, 2017. Association for Computing Machinery.

[62] Jack Nicas. Apple cracks down on apps that fight iPhone addiction. New York Times, 2019. https://www. nytimes.com/2019/04/27/technology /apple-screen-time-trackers.html?partner=rss\&emc=rss, accessed February 2020.

[63] Ofcom. Online nation 2019 report. Technical report, Ofcom, 2019 https://www.ofcom.org.uk/_data/assets/pdf_file/0024/ 149253/online-nation-summary.pdf, accessed January 2020.

[64] Fabian Okeke, Michael Sobolev, Nicola Dell, and Deborah Estrin. Good vibrations: Can a digital nudge reduce digital overload? In Proceedings of the 20th International Conference on Human-Computer Interaction with Mobile Devices and Services, MobileHCI '18, pages 4:1-4:12, New York, NY, USA, 2018. ACM.

[65] W. Oliveira, R. Oliveira, and F. Castor. A study on the energy consumption of Android app development approaches. In 2017 IEEE/ACM 14th International Conference on Mining Software Repositories (MSR), pages 42-52, 2017.

[66] Stack Overflow. How can $i$ tell if the screen is on in android? https://stackoverflow.com/questions/2474367/how-cani-tell-if-the-screen-is-on-in-android, accessed September 2019.

[67] Stack Overflow. Latest update on enabling and disabling mobile data programmatically. https://stackoverflow.com/questions/31120082/ latest-update-on-enabling-and-disabling-mobile-dataprogrammatically, accessed September 2019.

[68] Stack Overflow. Listing all installed apps on iPhone Programmatically? https://stackoverflow.com/questions/15332369/listin g-all-installed-apps-on-iphone-programmatically, accessed February 2020

[69] Stack Overflow. Simulate low bandwidth in android. https: //stackoverflow.com/questions/8693117/simulate-lowbandwidth-in-android, accessed September 2019.

[70] Arielle Pardes. Apple's restrictions aren't helping tech addiction. Wired 2018. https://www.wired.com/story/ios-restrictions-digita 1-wellness/, accessed February 2020.

[71] Sarah Perez. Apple puts third-party screen time apps on notice. Tech Crunch, 2018. https://techcrunch.com/2018/12/05/apple-puts-thirdparty-screen-time-apps-on-notice/, accessed February 2020

[72] James Pierce. Undesigning technology: Considering the negation of design by design. In Proceedings of the SIGCHI Conference on Human Factors in Computing Systems, CHI '12, pages 957-966, New York, NY, USA, 2012. ACM.

[73] Chris Preist, Daniel Schien, and Eli Blevis. Understanding and mitigating the effects of device and cloud service design decisions on the environmental footprint of digital infrastructure. In Proceedings of the 2016 CHI Conference on Human Factors in Computing Systems, CHI '16, pages 1324-1337, New York, NY, USA, 2016. ACM.
[74] Chris Preist, Daniel Schien, and Paul Shabajee. Evaluating sustainable interaction design of digital services: The case of YouTube. In Proceedings of the 2019 CHI Conference on Human Factors in Computing Systems, CHI '19, pages 397:1-397:12, New York, NY, USA, 2019. ACM.

[75] Quora. Is it possible for an ios app to programmatically turn off the phone's wifi? https://www. quora.com/Is-it-possible-for-an-ios-appto-programmatically-turn-off-the-phones-wi-fi, accessed February 2020.

[76] Ameet Ranadive and David Ginsberg. New tools to manage your time on facebook and instagram. Facebook, 2018. https://about.fb.com/news/ 2018/08/manage-your-time/, accessed January 2020

[77] Vemana Venkateswara Rao and A. S. N. Chakravarthy. Survey on Android forensic tools and methodologies. 2016

[78] Christian Reyter and Simon Scholl. Technical limitations for designing applications for social media. In Mensch \& Computer 2014 - Workshopband, Fourteenth Interdisciplinary Conference for Interactive and Cooperative Media - Interactivity on the Go, Berlin, Germany, 2014. De Gruyter Oldenbourg.

[79] Shannon Rodgers, Bernd Ploderer, Brittany Maloney, and Jason Hang. Designing for wellbeing-as-interaction. In Extended Abstracts of the 2019 CHI Conference on Human Factors in Computing Systems, CHI EA '19, pages LBW1817:1LBW1817:6, New York, NY, USA, 2019. ACM

[80] Android source. Data Saver mode. https://source. android.com/dev ices/tech/connect/data-saver, accessed September 2019.

[81] Stack Exchange. Android Enthusiasts: Block apps from accessing the Internet on Android device. https://android.stackexchange.com/ques tions/40924/block-apps-from-accessing-the-internet-onandroid-device, accessed September 2019.

[82] Stack Exchange. Android Enthusiasts: How to limit/throttle WiFi data speed on my Android device? https://android.stackexchange.com/questi ons/53890/how-to-limit-throttle-wifi-data-speed-on-myandroid-device, accessed September 2019.

[83] Stack Overflow. How to tell if 'Mobile Network Data' is enabled or disabled (even when connected by WiFi)? https://stackoverflow. com/ques tions/12806709/how-to-tell-if-mobile-network-data-isenabled-or-disabled-even-when-connected, accessed February 2020.

[84] Stack Overflow. is it possible to turn off wifi or switch iphone to offline mode in codes in swift 4 ? https://stackoverflow.com/question s/48616053/is-it-possible-to-turn-off-wifi-or-switchiphone-to-offline-mode-in-codes-in-swi, accessed February 2020 .

[85] Statista. Global market share held by smartphone operating systems from 2009 to 2017, 2019. https://www. statista.com/statistics/263453/glob al-market-share-held-by-smartphone-operating-systems/, accessed February 2020.

[86] StayFocusd, 2019. A tool for encouraging work focus and productivity: http: //www. stayfocusd.com/, accessed January 2020.

[87] Paul Thurrott. Apple's explanation for removing rival wellness apps is "misleading". Thurrott, 2019. https://www. thurrott.com/apple /206100/apples-explanation-for-removing-rival-wellnessapps-is-misleading\#, accessed February 2020.

[88] Ida Torres. Google further improves digital wellbeing initiatives for android devices. Android Community, 2019. https://androidcommunity.com /google-further-improves-digital-wellbeing-initiativesfor-android-devices-20190516/, accessed January 2020.

[89] Vincent W.-S. Tseng, Matthew L. Lee, Laurent Denoue, and Daniel Avrahami. Overcoming distractions during transitions from break to work using a conversational website-blocking system. In Proceedings of the 2019 CHI Conference on Human Factors in Computing Systems, CHI '19, New York, NY, USA, 2019. Association for Computing Machinery.

[90] Cold Turkey, 2019. An application for blocking websites: https://getcol dturkey.com/, accessed January 2020.

[91] Max Van Kleek, Reuben Binns, Jun Zhao, Adam Slack, Sauyon Lee, Dean Ottewell, and Nigel Shadbolt. X-Ray refine: Supporting the exploration and refinement of information exposure resulting from smartphone apps. In Proceedings of the 2018 CHI Conference on Human Factors in Computing Systems, CHI '18, pages 393:1-393:13, New York, NY, USA, 2018. ACM

[92] Pascal Welke, Ionut Andone, Konrad Blaszkiewicz, and Alexander Markowetz. Differentiating smartphone users by app usage. In Proceedings of the 2016 ACM International foint Conference on Pervasive and Ubiquitous Computing, UbiComp '16, page 519-523, New York, NY, USA, 2016. Association for Computing Machinery.

[93] Ashley Whillans. Our smartphone addiction is killing us - can apps that limit screen time offer a lifeline? The Conversation US, 2019. http://theconversation. com/our-smartphone-addictionis-killing-us-can-apps-that-limit-screen-time-offer-alifeline-116220, accessed January 2020 . 
[94] Kelly Widdicks. Understanding and Mitigating the Impact of Internet Demand in Everyday Life. PhD thesis, School of Computing and Communications, 2020.

[95] Kelly Widdicks, Oliver Bates, Mike Hazas, Adrian Friday, and Alastair R. Beresford. Demand around the clock: Time use and data demand of mobile devices in everyday life. In Proceedings of the 2017 CHI Conference on Human Factors in Computing Systems, CHI '17, pages 5361-5372, New York, NY, USA, 2017. ACM

[96] Kelly Widdicks, Mike Hazas, Oliver Bates, and Adrian Friday. Streaming, multiscreens and YouTube: The new (unsustainable) ways of watching in the home. In Proceedings of the 2019 CHI Conference on Human Factors in Computing Systems, CHI '19, pages 466:1-466:13, New York, NY, USA, 2019. ACM.

[97] Kelly Widdicks and Daniel Pargman. Breaking the Cornucopian Paradigm Towards moderate internet use in everyday life. In Proceedings of the Fifth Workshop on Computing Within Limits, LIMITS '19, pages 2:1-2:8, New York, NY, USA, 2019. ACM.
[98] Primal Wijesekera, Joel Reardon, Irwin Reyes, Lynn Tsai, Jung-Wei Chen, Nathan Good, David Wagner, Konstantin Beznosov, and Serge Egelman. Contextualizing privacy decisions for better prediction (and protection). In Proceedings of the 2018 CHI Conference on Human Factors in Computing Systems, CHI '18, pages 268:1-268:13, New York, NY, USA, 2018. ACM.

[99] Yous. How to check and toggle $\mathrm{WiFi}$ or $3 \mathrm{G} / 4 \mathrm{G}$ state in Android, 2013. https: //yous.be/2013/12/07/how-to-check-and-togglewifi-or-3g-4g-state-in-android/, accessed September 2019.

[100] Sha Zhao, Julian Ramos, Jianrong Tao, Ziwen Jiang, Shijian Li, Zhaohui Wu, Gang Pan, and Anind K. Dey. Discovering different kinds of smartphone users through their application usage behaviors. In Proceedings of the 2016 ACM International foint Conference on Pervasive and Ubiquitous Computing, UbiComp '16, pages 498-509, New York, NY, USA, 2016. ACM. 